\section{Falla de la profilaxis con cotrimoxazol para la neumonía por Pneumocystis jiroveci con el uso concomitante de leucovorina. Reporte de un caso}

\author{
Macarena Bonacic, Alfredo Silva y Marcelo Wolff
}

Failure of cotrimoxazole prophylaxis for Pneumocystis jiroveci pneumonia with concomitant use of leucovorin. Case report

The concomitant use of leucovorin and cotrimoxazole in PCP can lead to therapeutic failure and increased risk of death due to antagonism. It is important to keep this possible antagonistic interaction in mind even during prophylaxis. This paper presents a case with this failure outcome.

Key words: Leucovorin, folinic acid, Pneumocystis jiroveci, HIV prophylaxis, trimethoprim-sulfamethoxazole.

Palabras clave: Leucovorina, ácido folínico, Pneumocystis jiroveci, profilaxis VIH, trimetoprim/sulfametoxazol, cotrimoxazol.

\section{Introducción}

$\mathrm{S}$ e ha comunicado que el uso concomitante de leucovorina (ácido folínico) y cotrimoxazol puede producir mala respuesta de este último al tratamiento de la neumonía por Pneumocystis jiroveci aumentando el riesgo de fracaso y mortalidad ${ }^{1}$, por lo que se puede considerar como recomendación evitar la co-administración de leucovorina si se usa cotrimoxazol en forma terapéutica.

\section{Caso clínico}

Paciente de 25 años, sin antecedentes mórbidos. Consultó por una baja de peso no cuantificada de cuatro meses de evolución. Un mes antes del ingreso comenzó con odinofagia y disfagia confirmándose una candidiasis orofaríngea y esofágica por endoscopia. No recibió tratamiento específico y se procedió a investigar una posible infección por VIH, la que posteriormente se confirmó. Al examen físico destacaba un enflaquecimiento marcado y lesiones compatibles con candidiasis orofaríngea. Se encontraba afebril, eupneico, con examen pulmonar y radiografía de tórax normal.

Inició profilaxis con cotrimoxazol $960 \mathrm{mg}$ al día, ácido folínico $15 \mathrm{mg}$ al día, fluconazol $150 \mathrm{mg}$ al día y terapia anti-retroviral (TAR) con abacavir/

Hospital San Borja Arriarán, Unidad de Infectología (MB, MW).

Universidad de Chile, Facultad de Medicina.

Programa de formación en Medicina Interna (AS)

Conflictos de interés: Los autores declaran no tener conflictos de intereses.

Recibido: 31 de julio de 2012 / Aceptado: 6 de septiembre de 2012

Correspondencia a:

Macarena Bonacic

macabonacic@yahoo.com lamivudina y efavirenz, con muy buena tolerancia y adherencia. Dos semanas más tarde consultó por tres días de fiebre hasta $39^{\circ} \mathrm{C}$, cefalea y tos seca. Se decidió su internación por fiebre de $39^{\circ} \mathrm{C}$, taquicardia y saturación de oxígeno de $93 \%$ con aire ambiental. En el examen físico no había evidencia de candidiasis oral y el examen pulmonar en esos momentos era normal. La radiografía de tórax mostró un infiltrado algodonoso difuso bilateral. Los cultivos de expectoración, urocultivo, hemocultivos y baciloscopias resultaron negativos. La RPC para Pneumocystis jiroveci en una muestra de expectoración inducida fue negativa. Se ajustó el cotrimoxazol de dosis profiláctica a terapéutica por vía iv, se agregó prednisona y ceftriaxona iv, se suspendió el ácido folínico y se mantuvo la TAR iniciada recientemente.

A las 48 h, el paciente evolucionó afebril, con saturación de oxígeno adecuada y con mejoría de los parámetros inflamatorios. Se dio el alta hospitalaria al quinto día con cotrimoxazol oral y prednisona hasta completar 21 días. En el control ambulatorio estaba completamente asintomático y la radiografía mostró la desaparición del infiltrado pulmonar.

\section{Discusión}

Cotrimoxazol es la profilaxis de elección para la neumonía por P. jiroveci. Saah ${ }^{2}$ publicó los predictores de falla en la profilaxis, destacando la inmunosupresión grave (recuento de $\mathrm{CD} 4<85 \mathrm{cél} / \mathrm{mm}^{3}$ ) con un promedio de falla anual de $17,4 \%(\mathrm{p}<0,001)$ y como predictor clínico significativo, la fiebre y fatiga, con falla promedio anual de $21,3 \%(\mathrm{p}<0,05)$ y $16,5 \%$ $(\mathrm{p}<0,03)$; respectivamente. La causa más frecuente de falla en la profilaxis (95\%), es la mala adherencia a la medicación ${ }^{3,4}$. En este caso, el cuadro clínico y la respuesta terapéutica fue altamente sugerente de una neumonía por $P$. jiroveci pesar de la RPC negativa, lo que es frecuente con muestras de esputo inducido. Pneumocystis jiroveci es capaz de realizar síntesis de novo de ácido fólico ${ }^{5}$ lo que explicaría que la administración exógena de ácido folínico permita esta síntesis y antagonice el efecto antimicrobiano de cotrimoxazol frente a este agente. En este caso la concomitancia de administración de ácido folínico con cotrimoxazol parece haber tenido precisamente este efecto. En conclusión, el uso de leucovorina debe reservarse para pacientes seleccionados y debe utilizarse con extrema precaución cuando se co-administra con cotrimoxazol como profilaxis de neumonía por $P$. jiroveci.

\section{Referencias bibliográficas}

1.- Safrins S, Lee BL, Sande M A. Adjunctive folinic acid with $\mathrm{t}$ imethoprim-sulfamethoxazole for Pneumocystis carinii pneumonia in AIDS patients is associated with an increased risk of therapeutic failure and death. J Infect Dis 1994; 170: 912-7.

2.- Saah A J, Hoover D R, Peng Y, Phair JP, Visscher B, Kingsley L A, et al. Predictors for failure of Pneumocystis carinii pneumonia prophylaxis. Multicenter AIDS Cohort Study. JAMA 1995; 273: 1197-202.

3.- Bonora S, Di Perri G, Vento S, Cazzadori A, Concia E. Failure of prophylaxis against PCP in patients with HIV infection. AIDS Patient Care STDS 1998;12: 843-8.

4.- Lundberg B E, Davidson AJ, Burman W J. Epidemiology of Pneumocystis carinii pneumonia in an era of effective prophylaxis: the relative contribution of non-adherence and drug failure. AIDS 2000; 14: 2559-66.

5.- Kaneshiro E S. Pneumocystis carinii pneumonia: the status of Pneumocystis biochemistry. Int J Parasitol 1998; 28: 65-84. 